\title{
A prospective observational study of mycophenolate mofetil treatment in progressive diffuse cutaneous systemic sclerosis of recent onset.
}

\author{
Fabian A. Mendoza, MD \\ Thomas Jefferson University, Fabian.MendozaBallesteros@jefferson.edu \\ Sarah J. Nagle \\ Thomas Jefferson University, Sarah.Nagle@jefferson.edu \\ Jason B. Lee, MD \\ Thomas Jefferson University, jason.lee@jefferson.edu \\ Serioio A Jimenez \\ Thomas Jefferson University, Sergio.Jimenez@jefferson.edu \\ Part of the Dermatology Commons, and the Rheumatology Commons \\ Let us know how access to this document benefits you
}

\section{Recommended Citation}

Mendoza, MD, Fabian A.; Nagle, Sarah J.; Lee, MD, Jason B.; and Jimenez, Sergio A., "A

prospective observational study of mycophenolate mofetil treatment in progressive diffuse

cutaneous systemic sclerosis of recent onset." (2012). Jefferson Institute of Molecular

Medicine Papers and Presentations. Paper 12.

https://jdc.jefferson.edu/jimmfp/12

This Article is brought to you for free and open access by the Jefferson Digital Commons. The Jefferson Digital Commons is a service of Thomas Jefferson University's Center for Teaching and Learning (CTL). The Commons is a showcase for Jefferson books and journals, peer-reviewed scholarly publications, unique historical collections from the University archives, and teaching tools. The Jefferson Digital Commons allows researchers and interested readers anywhere in the world to learn about and keep up to date with Jefferson scholarship. This article has been accepted for inclusion in Jefferson Institute of Molecular Medicine Papers and Presentations by an authorized administrator of the Jefferson Digital Commons. For more information, please contact: JeffersonDigitalCommons@jefferson.edu. 


\section{A prospective observational study of mycophenolate mofetil treatment in progressive diffuse cutaneous Systemic Sclerosis of recent onset.}

Fabian A. Mendoza, MD ${ }^{1,2}$, Sarah J. Nagle ${ }^{3}$, Jason B. Lee, $\mathrm{MD}^{4}$ and Sergio A. Jimenez, MD 1

1 Jefferson Institute of Molecular Medicine and Scleroderma Center, Thomas Jefferson University

2 Rheumatology Division, Department of Medicine, Thomas Jefferson University

3 Jefferson Medical College, Thomas Jefferson University

${ }^{4}$ Dermopathology Division, Department of Dermatology and Cutaneous Biology, Thomas Jefferson University.

Keywords: Systemic Sclerosis, Scleroderma, Treatment, Mycophenolate Mofetil, Fibrosis

\section{Corresponding Author}

Sergio A. Jimenez, M.D.

Jefferson Institute of Molecular Medicine

Thomas Jefferson University

233 S. 10 ${ }^{\text {th }}$ Street, Suite 509 BLSB

Philadelphia, PA 19107. USA.

T: 215-503-5042 / F: 215-923-4649

Sergio.Jimenez@jefferson.edu 
Objective: Prospective observational study of mycophenolate mofetil (MMF) treatment in patients with diffuse progressive cutaneous Systemic Sclerosis (SSc) of recent onset.

Methods: Twenty five previously untreated consecutive patients with recent onset ( $<24$ months) diffuse progressive cutaneous SSc received MMF as the only disease-modifying therapy. Modified Rodnan skin score (mRSS) and affected body surface area (BSA) were compared from initiation of MMF to study end. Pulmonary function tests performed at the same institution before therapy and at study end were available in 15 patients. Histopathology and real time PCR assessment of fibrosis-related gene expression were performed before and after treatment in skin biopsies from 3 patients.

Results: At 18.2 \pm 8.73 months of MMF therapy (median $2000 \mathrm{mg} /$ day) the mRSS decreased from $24.56 \pm 8.62$ to $14.52 \pm 10.9(\mathrm{p}=0.0004)$ and the affected BSA from $36 \pm 16 \%$ to $14 \pm 13.3 \%$ $(\mathrm{p}=0.00001)$. Pulmonary function tests remained stable from initiation of MMF to the end of the study. Skin histopathology showed a remarkable reduction in fibrotic tissue accumulation. Real time PCR of skin biopsies demonstrated a marked decrease in expression of fibrosis-related genes.

Conclusion: Patients with diffuse progressive cutaneous SSc of recent onset treated with MMF experienced marked improvement in skin involvement and stabilization of pulmonary function. Skin biopsies from 3 patients demonstrated histopathological improvement and decreased expression of fibrosis-related genes. 


\section{Introduction}

Systemic sclerosis ( $\mathrm{SSc}$ ) is a heterogeneous autoimmune disease characterized by increased collagen deposition in skin and multiple internal organs, prominent vascular and endothelial abnormalities and humoral and cellular immune dysregulation (1-3). The introduction of target organ specific therapies has substantially decreased Systemic Sclerosis (SSc) morbidity and mortality. In contrast, most studies of potential disease-modifying agents have shown negative results and currently there is no generally accepted SSc disease modifying therapy.

A major challenge for the evaluation of effectiveness of therapeutic interventions in SSc are the heterogeneity and variable course of the disease. To mitigate the effects of SSc course variability and following the paradigm that early intervention is crucial for halting fibrosis most recent studies selected patients with recent onset of disease (5-9). Disease of recent onset has been defined in different ways in various studies although a cut-off point of 18-24 months from the appearance of clinically detectable skin induration is generally accepted. Furthermore, patients should have substantial skin involvement at treatment initiation to allow detection of improvement employing the outcome measure tools currently available.

Mycophenolate mofetil (MMF) is an immunosuppressant agent that inhibits proliferative responses of $\mathrm{T}$ and $\mathrm{B}$ lymphocytes as well as antibody production by $\mathrm{B}$ lymphocytes. It is indicated for prevention of organ rejection following allogeneic cardiac, hepatic and renal transplants (10). It is also used for induction and maintenance therapy of lupus nephritis $(11,12)$. Furthermore, there is evidence that MMF can inhibit fibrosis by acting either directly on fibroblasts (13) or by inhibiting fibroblast proliferation (14), and by reducing tissue accumulation of activated myofibroblasts (15). 
Several studies including a recent assessment of 98 patients have described encouraging results of MMF treatment for SSc (16-20). In contrast, a recent observational trial failed to show significant differences between four different therapeutic approaches, three of which included MMF (21). Here, we describe a prospective observational study of MMF for the treatment of SSc in patients with recent onset of progressive diffuse cutaneous involvement. In contrast with other published studies (16-20) the patients included in our study had not received any previous immunosuppresive, immunomodulating, or antifibrotic therapy, and none of the patients received any other disease modifying therapy during the study.

\section{Patients and Methods}

Patient cohort. From December 2006 to December 2009, every new patient with diffuse and rapidly progressive SSc of recent onset evaluated at our institution was offered treatment with MMF as disease modifying monotherapy. Only patients with less than 2 years of disease and mRSS of greater than 12 points who had not received any previous immunosuppressive, immunomodulatory, or anti-fibrotic therapy were included. Despite the consecutive enrollment design not all qualifying patients received MMF owing to diverse reasons including most commonly the denial of insurance reimbursement.

All patients received 500mg twice a day MMF for 3 to 4 weeks and then the dose was escalated to 1-1.5 g twice a day. Other concomitant medications included calcium channel blockers, angiotensin converting enzyme inhibitors, proton pump inhibitors, statins and low dose aspirin. The effects of MMF on the extent and severity of skin involvement employing the modified Rodnan Skin Score (mRSS) and an estimate of extent of affected body surface area (BSA) were 
assessed as described previously (22). The effects of MMF on SSc severity employing some of the categories of Medsger's severity scale (23) were also examined.

The inclusion criteria were: (i) time from onset of clinically detectable skin induration to initiation of MMF less than 24 months; (ii) diffuse skin involvement at initiation of MMF affecting the trunk and /or arms and legs proximally to the elbows and /or knees with a mRSS greater than 12; (iii) no previous treatment with MMF, D-penicillamine, bovine collagen, cyclophosphamide or any other putative SSc disease modifying drug or intervention; and (iv) the presence of progressive skin involvement based on information provided by the referring rheumatologist or by sequential examination at our center.

The exclusion criteria were: (i) skin involvement confined to face or acral regions of the body (limited cutaneous SSc); (ii) Eosinophillic fasciitis, Nephrogenic Systemic Fibrosis, or other scleroderma -like diseases; (iii) Mixed connective tissue disease and overlap syndromes; (iv) prior or current therapy with any other immunosupressant or antifibrotic agent and (v) poor compliance to medications or follow up.

Study entry was the initiation of MMF treatment and end of study was the last recorded visit. For data analysis only patients who were followed up for at least 6 months were included.

Assessment of MMF effects on cutaneous and visceral involvement. The extent and severity of skin involvement was assessed by the mRSS, and the extent of affected BSA was assessed by the rule of nines employing a burns victim diagram as described previously (22). These parameters were recorded every 3-6 months during the treatment follow up. Pulmonary function tests, including total lung capacity (TLC) and diffusion capacity for carbon monoxide (DLCO) were 
monitored every 6 to 12 months during the study. Although chest computerized tomography (CT) is part of the evaluation and management for all SSc patients at our center CT findings were not systematically followed up. Percentages and means \pm SD were used to describe the cohort's demographics. A paired two-tail Student's t test was used to compare data during the study period. Because skin involvement continues to increase in extent and severity for a variable length of time following initiation of therapy in patients with diffuse SSc of recent onset comparisons of skin involvement were performed between the initial vs final scores and between the maximal (peak) vs final scores.

Effects of MMF on skin histopathology and fibrosis-related gene expression. Pre- and posttreatment skin biopsies were performed in three patients with their informed consent. Punch biopsy samples were obtained from affected skin in the dorsum of the forearm. The posttreatment samples were from an area removed approximately $5 \mathrm{~mm}$ from the initial skin biopsy. Histopathology assessment was performed in hematoxillin eosin stained skin biopsy samples. Total RNA was extracted from all the biopsies using RNeasy Fibrous Tissue Kit (Qiagen, Valencia, CA). Each sample was retro-transcribed using SuperScript II reverse transcriptase (Invitrogen) and real-time quantitative PCR (rt-PCR) was performed for the expression of COL1A1, COL1A2, COL4A1, COL14A1, CTGF, FN1, $\alpha$ SMA and TGF- $\beta 1$. Sequence specific primers (Table 1) were designed using PrimerQuest (Integrated DNA Technologies, Coralville, IA). Rt-PCR was performed using SYBR green with an ABI Prism 7900 system (Applied Biosystems, Carlsbad, CA). The mRNA expression level was normalized by 18 s ribosomal RNA. 


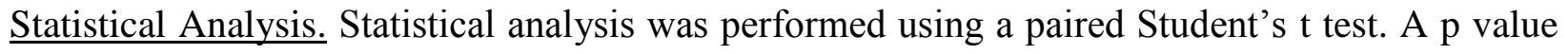
of $<0.05$ was considered statistically significant. Statistical analyses were performed using Excel and GraphPad Prism programs.

\section{Results}

Demographics. There were 35 patients with progressive SSc who fulfilled the classification criteria for SSc of the American College of Rheumatology (24) and the criteria of LeRoy et al. (25) for the clinical subset of diffuse cutaneous SSc. All patients had disease of recent onset and had not received any prior immunomodulatory or immunosuppressive therapy nor any putative anti-fibrotic agent or intervention. Twenty five patients met the inclusion and exclusion criteria and were followed at our institution for at least 6 months. The mean \pm SD age at initiation of treatment was $48.6 \pm 14.5$ years. The majority of the patients were caucasians $(76 \%)$ with a smaller proportion of African Americans (12\%). The autoantibody profile indicated that $100 \%$ had a positive antinuclear antibody (ANA), by immunofluorescence and 36\% (9 patients) harbored anti-Scl-70 antibodies. The remaining patients were ANA positive but Scl-70 and anticentromere antibody negative. Analysis for RNA polymerase I/III or other SSc specific antibodies were not performed in all patients studied. The average duration of therapy was $18.2 \pm$ 8.73 months, and 18 out of $25(72 \%)$ were treated for longer than 1 year. The average dose of MMF was $2.02 \mathrm{~g} /$ day. Only 3 patients had received less than $2 \mathrm{~g} / \mathrm{d}$ owing to gastrointestinal side effects.

Clinical assessment of skin involvement and pulmonary function. Clinically detectable skin induration was first noted at a mean $\pm \mathrm{SD}$ of $14.1 \pm 7.3$ months prior to study entry. At the initiation of therapy the mRSS was $24.56 \pm 8.62$ units and the affected BSA was $36 \pm 16 \%$. In the vast majority of patients a worsening of mRSS and extent of affected BSA was noted 
following entry into the study reaching a peak from 3-6 months after initiation of MMF therapy. The average time from the first detectable skin involvement to the maximal involvement (considered as the maximal mRSS) was 20.1 months and the interval between initiation of treatment to peak mRSS was 6.4 months. Maximal mean \pm SD mRSS and BSA were $27.36 \pm$ 9.71 points and $38 \pm 15.4 \%$, respectively. The increase in mRSS from the initiation of therapy to maximal involvement was statistically significant $(\mathrm{p}=0.0065)$. Although the increase in BSA value displayed a similar trend it did not reach statistical significance. $(\mathrm{p}=0.069)$. A significant reduction of the mRSS and affected BSA was noted from the beginning of therapy to the end of the study. The mRSS decreased from $24.56 \pm 8.62$ at entry to $14.52 \pm 10.9(\mathrm{p}=0.004)$ and the BSA decreased from $36 \pm 16 \%$ at entry to $14 \pm 13.3 \%$ ( $\mathrm{p}=0.00001$ ). A more dramatic change was observed when the peak involvement was compared with the involvement at the end of the study. The values observed were $27.36 \pm 9.7$ vs $14.52 \pm 10.9\left(\mathrm{p}=2.5 \times 10^{-6}\right)$ for the mRSS and 38 $\pm 15.4 \%$ vs $14 \pm 13.3 \%\left(\mathrm{p}=9.2 \times 10^{-7}\right)$.

Figure 1 shows the mRSS values and extent of BSA treatment initiation (point 1), at peak involvement (point 2), and at last follow up (point 3). The changes in affected BSA in three patients in whom skin biopsies were obtained before initiation of MMF therapy and at the end of the study are pictured in Figure 2. The modified Medsger Severity Score was assessed in all patients in the study, however, the values represent an underestimate of the severity of the disease since not all patients with gastrointestinal symptoms were examined by endoscopy or barium swallow and muscle involvement was not routinely evaluated. Scores for organ involvement measured by the Medsger Severity Score at initiation of treatment compared with the scores at study end did not reach statistical significance with the exception of the skin index that demonstrated a significant reduction $(2.2 \pm 0.71$ versus $1.52 \pm 0.77, \mathrm{p}=0.0003)$. 
The results of pulmonary function tests were analyzed in $15 / 25$ patients who had pre- and posttreatment pulmonary function tests performed at the same institution and were, therefore, consistent in methodology and equipment utilized. The results in these patients showed that TLC was $89.47 \pm 16.61 \%$ of predicted at study entry compared with $85.33 \pm 17.34 \%$ at study end $(\mathrm{p}=0.13)$. In this subgroup of $15 / 25$ patients only 3 patients showed a decrease in their TLC values greater than $10 \%$. The results for DLCO were $69.0 \% \pm 17.51 \%$ of predicted at study entry compared to $70.47 \% \pm 18.84 \%$ of predicted at study end $(\mathrm{p}=0.45)$.

Tolerability and Adverse Events. All patients tolerated MMF treatment well and in no case was MMF discontinued owing to intolerance or side effects although in 3 of the 25 patients studied the dose was reduced to $1-1.5 \mathrm{~g} /$ day because of gastrointestinal side effects. Ten patients developed at least one adverse event during MMF treatment. A total of 13 events were recorded. The most common events were diarrhea (3) and upper respiratory infections (3), followed by lymphopenia (2), and urinary tract infection (2). One of these 10 patients required a dose adjustment because of leukopenia. One of the patients in the study died at 18 months of entry. The cause of dead was a severe and rapidly progressive dilated cardiomyopathy of unkown etiology. Although it is very unlikely that this event was related to MMF treatment the possibility that it represented an abrupt deterioration of SSc cardiac involvement or a viral infection possibly related to immunosupression can not be conclusively established. However, at her last evaluation following 9 months of MMF therapy and approximately 10 months before her demise there was a clear reduction in both mRSS and total BSA thus, rendering the possibility that her demise was SSc related less likely.

$\underline{\text { Skin histopathological analysis and assessment of fibrosis-related gene expression. }}$ 
Histopathological examination and analysis of fibrosis-related gene expression were performed in pre- and post-treatment skin biopsies obtained from 3 patients. As illustrated in Figure 3, hematoxyllin-eosin staining showed a remarkable decrease in the abundance and thickness of collagen bundles and in their compact appearance in the dermis. Similar findings were observed in skin biopsies stained with Masson's Trichrome (not shown). There was also very noticeable re-appearance of hair follicles and sweat and sebaceous glands in the post-treatment biopsies. Analysis of relative gene expression by real time quantitative PCR, demonstrated a marked decrease in the relative expression of numerous fibrosis-related genes including COL1A1, COL1A2, COL 4A1, COL 14A1, CTGF, FN1, $\alpha$ SMA and TGF- $\beta 1$. (Figure 4).

\section{Discussion}

Mycophenolate mofetil, through its biologically active molecule, mycophenolic acid exerts potent antiproliferative properties resulting from the reversible inhibition of inosine monophosphate dehydrogenase (IMPDH). Given the higher sensitivity of the lymphocyte IMPDH isoform, MMF exerts its effects by causing preferential lymphocyte suppression. On the other hand, recent studies have shown that MMF is also able to decrease fibroblast proliferation, an effect which appears to be mediated mainly by IMPDH dependent pathways although IMPDH independent pathways can also play a role $(14,15)$. It also has been shown that MMF is capable of direct inhibition of collagen production (13).

The observed degree of improvement of mRSS in the study described here correlates well with prior studies by Derk et al. (19) and Le et al. (20) who found a reduction of 14.1 and 7.6 points in the mRSS of patients treated with MMF, respectively. Given the heterogeneous SSc course, the greater than 10 point mRSS improvement and the $23 \%$ reduction in affected BSA observed here are likely clinically significant for this population of previously untreated patients with SSc of 
recent onset with diffuse cutaneous involvement and usually progressive clinical course. Furthermore, these results are also superior to our previously published data on D-penicillamine in a similar population (22). These results are also superior to the spontaneous reduction in mRSS observed in the placebo control group (-4.6) and in the relaxin-treated group (-4.4) in a recently published study describing the results of a 24 week randomized control trial of human recombinant relaxin in a population of SSc patients similar to the one we studied here (26) and to the -3 point reduction at 15 months in either placebo or treated group in patients with early phase SSc entering the bovine type I collagen randomized control trial (27).

The clinical improvement we observed correlated well with a remarkable decrease in histopathological fibrosis, re-appearance of dermal hair follicles and sebaceous and sweat glands, as well as, with a marked reduction in the expression of numerous fibrosis-related genes in samples from three patients in whom skin biopsies were obtained before and after therapy. Of importance was the observation that MMF treatment prevented pulmonary function deterioration in this population with diffuse SSc of recent onset in whom substantial and significant deterioration of lung function is highly likely. However, it should be emphasized that the cohort studied here had only a mild baseline decrease in TLC at study entry, a variable which may have contributed to slow progression of pulmonary function deterioration as described by Plastiras et al. (28). The stabilization of lung involvement as assessed by comparison of pulmonary function tests from initiation of MMF treatment to the end of the study is similar to the results recently describe by Simeon-Aznar et al in 14 consecutive SSc patients who were treated with $1.5 \mathrm{~g}$ of MMF for 12 months (29).

Although the number of patients described here is not large, there are several strengths to our study including the prospective observational design of our study, and the very strict criteria used 
for defining the study population requiring that only patients with very early (less than 2 years) and progressive skin disease be included. This is in contrast with populations with much longer duration of skin disease described in the majority of other studies of SSc disease modifying interventions. Another strength of our study is that all patients included were naïve to any immunosupressants or anti-fibrotic treatment and received MMF as the only disease modifying therapy for the duration of the study.

The selection of patients with early disease was required in order to mitigate the effects of the variable course of SSc and to address the paradigm that early intervention is crucial for effective treatment in SSc and other fibrotic diseases. On the other hand, given the relatively low sensitivity of the current tools for disease assessment the inclusion of patients with moderate to severe disease was important for demonstrating differences induced by the therapeutic intervention.

From the safety perspective MMF related adverse events were mild and well tolerated and only three patients required a dose adjustment. Discontinuation of MMF was not necessary in any patient. In contrast to the benign pattern of MMF side effects other options for SSc disease modification, including cyclophosphamide and bone marrow transplant carry substantial and even fatal potential side effects $(30,31)$. Furthermore, the frequency and severity of adverse events we observed in this study are not greater than those found in patients with other autoimmune diseases or in renal transplant patients treated with MMF.

We are aware that owing to the non-placebo controlled design of our study it is not possible to draw definitive conclusions regarding the efficacy of MMF in modifying SSc disease course. However, the remarkable degree of clinical improvement observed in this cohort of progressive 
SSc of recent onset which was strongly corroborated by histopathological and gene expression studies is unlikely to be explained only by the natural evolution of the disease, particularly, in this group of patients with very early and progressive disease. Furthermore, given the benign safety profile of MMF our findings suggest that therapy with MMF is safe and effective for SSc patients with progressive diffuse cutaneous disease of recent onset.

Given the major limitations and substantial challenges in the study of potential SSc disease modifying treatment prospectively designed double-blind studies will be needed to identify effective therapeutic interventions for this often devastating disease. 


\section{Acknowledgements}

We gratefully acknowledge the technical assistance of Mrs. Megan Musick in the performance of the gene expression studies. The excellent assistance of Mrs. Melissa Bateman in the preparation of the manuscript is also gratefully acknowledged.

Supported in part by NIH Grant AM 19616 to Sergio A. Jimenez.

\section{Disclosures}

The authors do not have any competing or conflict of interest to disclose. 


\section{References}

1. Gabrielli A, Avvedimento EV, Krieg T Scleroderma .N Engl J Med. 2009;360(19):19892003

2. Wei J, Bhattacharyya S, Tourtellotte WG, Varga J: Fibrosis in systemic sclerosis: Emerging concepts and implications for targeted therapy. Autoimmun Rev. 2010 (in press)

3. Katsumoto TR, Whitfield ML, Connolly MK. The pathogenesis of systemic sclerosis. Annu Rev Pathol. 2011 Feb 28;6:509-37.

4. Steen VD, Costantino JP, Shapiro AP, Medsger TA Jr. Outcome of renal crisis in systemic sclerosis: relation to availability of angiotensin converting enzyme (ACE) inhibitors. Ann Intern Med 1990,113:352-357.

5. Clements PJ, Furst DE, Wong WK, Mayes M, White B, Wigley F, et al. High-dose versus low-dose D-penicillamine in early diffuse systemic sclerosis: analysis of a two-year, double-blind, randomized, controlled clinical trial. Arthritis Rheum 1999; 42:1194-1203.

6. Denton CP, Merkel PA, Furst DE, Khanna D, Emery P, Hsu VM, et al. Recombinant human anti-transforming growth factor beta1 antibody therapy in systemic sclerosis: a multicenter, randomized, placebo-controlled phase I/II trial of CAT-192. Arthritis Rheum 2007; 56:323-333.

7. Pope, JE, Bellamy N, Seibold JR, Baron M, Ellman M, Carette S, et al. A Randomized control trial of Methotrexate versus placebo in early diffuse Scleroderma. Arthritis Rheum 2001; 44:1351-1358.

8. van den Hoogen FH, Boerbooms AM, Swaak AJ, Rasker JJ, van Lier HJ, van de Putte LB. Comparison of methotrexate with placebo in the treatment of systemic sclerosis: a 24 week randomized double-blind trial, followed by a 24 week observational trial. Br J Rheumatol 1996; 35:364-372.

9. Black CM, Silman AJ, Herrick AI, Denton CP, Wilson H, Newman J, et al. Interferonalpha does not improve outcome at one year in patients with diffuse cutaneous scleroderma: results of a randomized, double-blind, placebo-controlled trial. Arthritis Rheum 1999; 42:299-305.

10. Cellcept prescribing information. Genentech Inc (Rev Feb 2010)

11. Appel GB, Contreras G, Dooley MA, Ginzler EM, Isenberg D, Jayne D, et al. Mycophenolate mofetil versus cyclophosphamide for induction treatment of lupus nephritis. J Am Soc Nephrol. 2009;20:1103-12.

12. Radhakrishnan J, Moutzouris DA, Ginzler EM, Solomons N, Siempos II, Appel GB. Mycophenolate mofetil and intravenous cyclophosphamide are similar as induction therapy for class V lupus nephritis. Kidney Int. 2010;77:152-60.

13. Roos N, Poulalhon N, Farge D, Madelaine I, Mauviel A, Verrecchia F. In vitro evidence for a direct antifibrotic role of the immunosuppressive drug mycophenolate mofetil. J Pharmacol Exp Ther 2007; 321:583-9. 
14. Petrova DT, Brandhorst G, Brehmer F, Gross O, Oellerich M, Armstrong VW. Mycophenolic acid displays IMPDH-dependent and IMPDH-independent effects on renal fibroblast proliferation and function. Ther Drug Monit 2010; 32:405-12.

15. Badid C, Vincent M, McGregor B, Melin M, Hadj-Aissa A, Veysseyre C, et al. Mycophenolate mofetil reduces myofibroblast infiltration and collagen III deposition in rat remnant history. Kidney Int. $2000 \mathrm{Jul} ; 58(1): 51-61$.

16. Vanthuyne M, Blockmans D, Westhovens R, Roufosse F, Cogan E, Coche E et al. A pilot study of mycophenolate mofetil combined to intravenous methylprednisolone pulses and oral low-dose glucocorticoids in severe early systemic sclerosis. Clin Exp Rheumatol 2007; 25:287-92.

17. Nihtyanova SI, Brough GM, Black CM, Denton CP. Mycophenolate mofetil in diffuse cutaneous systemic sclerosis--a retrospective analysis. Rheumatology (Oxford) 2007; 46:442-5.

18. Gerbino AJ, Goss CH, Molitor JA. Effect of mycophenolate mofetil on pulmonary function in scleroderma-associated interstitial lung disease. Chest 2008; 133:455-60.

19. Derk CT, Grace E, Shenin M, Naik M, Schulz S, Xiong W. A prospective open-label study of mycophenolate mofetil for the treatment of diffuse systemic sclerosis. Rheumatology (Oxford) 2009; 48:1595-9.

20. Le EN, Wigley FM, Shah AA, Boin F, Hummers LK. Long-term experience of mycophenolate mofetil for treatment of diffuse cutaneous systemic sclerosis. Ann Rheum Dis. 2011;70:1104-7.

21. Herrick AL, Lunt M, Whidby N, Ennis H, Silman A, McHugh N et al. Observational study of treatment outcome in early diffuse cutaneous systemic sclerosis. J Rheumatol 2010; 37:116-24.

22. Derk CT, Huaman G, Jimenez SA. A retrospective randomly selected cohort study of Dpenicillamine treatment in rapidly progressive diffuse cutaneous systemic sclerosis of recent onset Br J Dermatol. 2008; 158:1063-8.

23. Medsger TA Jr, Silman AJ, Steen VD, Black CM, Akesson A, Bacon PA et al. A disease severity scale for systemic sclerosis: development and testing. J Rheumatology 1999; 26:2159-67.

24. Preliminary criteria for the classification of systemic sclerosis (scleroderma). Subcommittee for scleroderma criteria of the American Rheumatism Association Diagnostic and Therapeutic Criteria Committee. Arthritis Rheum 1980; 23:581-590.

25. LeRoy EC, Black C, Fleischmajer R, Jablonska S, Krieg T, Medsger TA Jr, et al. Scleroderma (systemic sclerosis): classification, subsets and pathogenesis. J Rheumatol 1988; 15:202-205.

26. Khanna D, Clements PJ, Furst DE, Korn JH, Ellman M, Rothfield N, et al. Recombinant human relaxin in the treatment of systemic sclerosis with diffuse cutaneous involvement: a randomized, double-blind, placebo-controlled trial. Arthritis Rheum. 2009; 60:1102-11.

27. Postlethwaite AE, Wong WK, Clements P, Chatterjee S, Fessler BJ, Kang AH, et al. A multicenter, randomized, double-blind, placebo-controlled trial of oral type I collagen 
treatment in patients with diffuse cutaneous systemic sclerosis: I. oral type I collagen does not improve skin in all patients, but may improve skin in late-phase disease. Arthritis Rheum. 2008;58:1810-22.

28. Plastiras SC, Karadimitrakis SP, Ziakas PD, Vlachoyiannopoulos PG, Moutsopoulos HM, Tzelepiz GE. Scleroderma Lung: Initial Forced Vital Capcity as Predictor of Pulmonary Function Decline. Arthritis Rheum. 2006;55:598-602.

29. Simeón-Aznar CP, Fonollosa-Plá V, Tolosa-Vilella C, Selva-O'Callaghan A, Solans-Laqué $\mathrm{R}$, Vilardell-Tarrés M. Effect of mycophenolate sodium in scleroderma-related interstitial lung disease. Clin Rheumatol. 2011 Sep 1. [Epub ahead of print].

30. Furst DE, Tseng CH, Clements PJ, Strange C, Tashkin DP, Roth MD, et al. Adverse events during the Scleroderma Lung Study. Am J Med. 2011;124:459-67.

31. Binks M, Passweg JR, Furst DE, McSweeney P, Sullivan K, Besenthal C, et al. Phase I/II trial of autologous stem cell transplantation in systemic sclerosis: procedure related mortality and impact on skin disease. Ann Rheum Dis. 2001;60:577-84. 
Table 1: Sequence of primers utilized for real-time PCR

COL 1A1 Forward: CCTCAAGGGCTCCAACGAG

COL1A1 Reverse: TCAATCACTGTCTTGCCCCA

COL1A2 Forward: GGCAAACATGGAAACCGTGGTGAA

COL1A2 Reverse: GGCAGACCTTGCAATCCATTGTGT

COL4A1 Forward: TCCTCATTCTGCATCCTGGCTTGA

COL4A1 Reverse: AAATGGCCCGAATGTGCTTACGTG

COL11A1 Forward: TTGGTCTGCAGTCGCAATTTCGTG

COL11A1 Reverse: TGTTACGGTGAAATCCCAGAGCCA

COL14A1 Forward: AGTCTGGATGGCCACCTACAACAA

COL14A1 Reverse: TTGGTGACCACCGCATGAAGTTTG

CTGF Forward: CTGCAGgCTAGAGAAGCAGAG

CTGF Reverse: TTGCCCTTCTTAATGTTCTCTTCC

FN-1 Forward: TTGATGCCGTTTCCAGCCAAT

FN-1 Reverse: AAACGCAGGTTGGATGGTGCAT

aSMA Forward: TGTATGTGGCTATCCAGGCG

aSMA Reverse: AGAGTCCAGCACGATGCCAG

TGF-ק1 Forward: CGAGCCTGAGGCCGACTA

TGF- $\boldsymbol{\beta 1}$ Reverse: AGATTTCGTTGTGGGTTTCCA 


\section{Figure 1}

Assessment of mRSS. Assessment was performed, at initiation of treatment (point 1), at peak of skin involvement (point 2), and at last follow up (point 3).

\section{Figure 2}

Assessment of total body surface involvement (BSA) in three patients who had skin biopsies

before and after MMF treatment. (A) BSA at the initiation of MMF therapy. (B-D) BSA at serial follow up evaluations (approximately every three months).

\section{Figure 3}

Histopathology of pre treatment (A and C) and post treatment (B and D) hematoxylin-eosin stained skin biopsies. Images are at 100x magnification. A and B show epidermis and upper dermis and $\mathrm{C}$ and $\mathrm{D}$ show lower dermis. Note the remarkable decrease in the thickness and compact appearance of the collagen bundles, as well the re-population of dermal structures following MMF treatment.

\section{Figure 4}

Relative change in Real Time PCR fibrosis-related gene expression from beginning to end of treatment in skin biopsy samples from 3 patients. Measurement of various collagen genes (COL1A1, COL1A2, COL4A1, COL11A1, COL14A1), connective tissue growth factor (CTGF), fibronectin 1 (FN-1), alpha smooth muscle actin ( $\alpha \mathrm{SMA})$ and transforming growth factor beta (TGF- $\beta$ ) are shown. The horizontal scale shows expression levels (\%). Pre-treatment levels were normalized to $100 \%$. 
Figure 1

mRSS

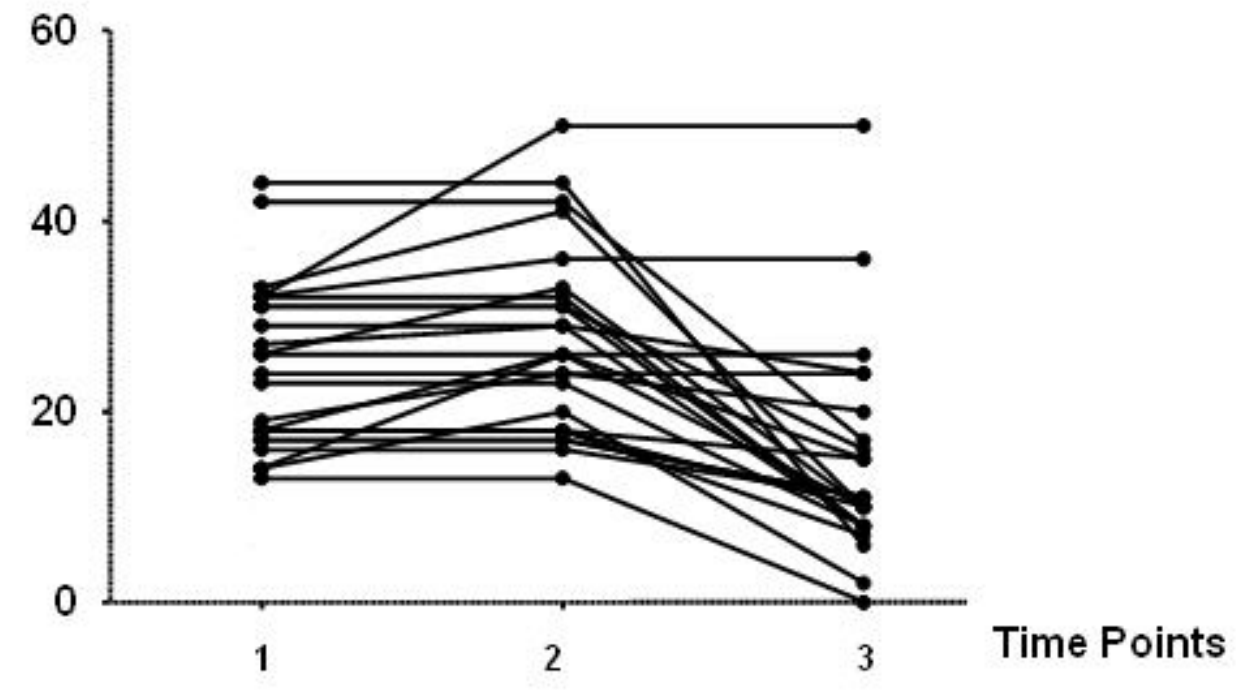

Figure 1A

\section{BSA $\%$}

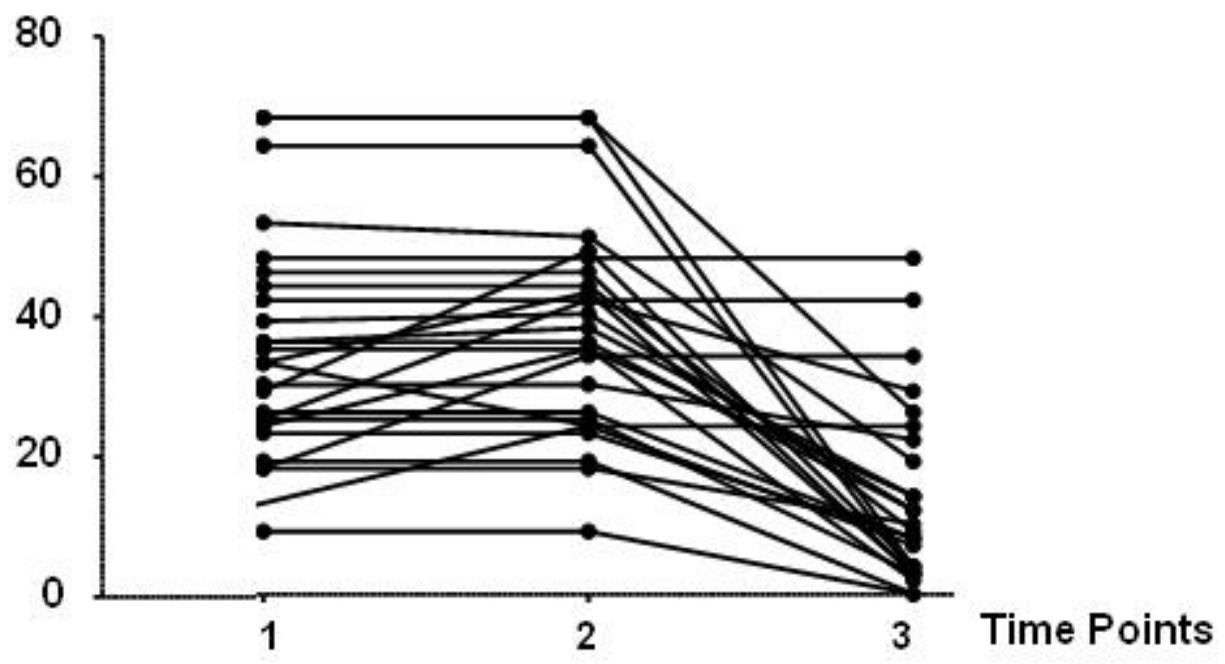

Figure 1B 
Before Treatment

A
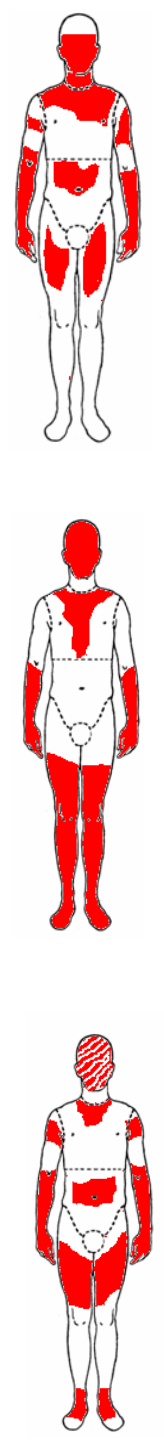
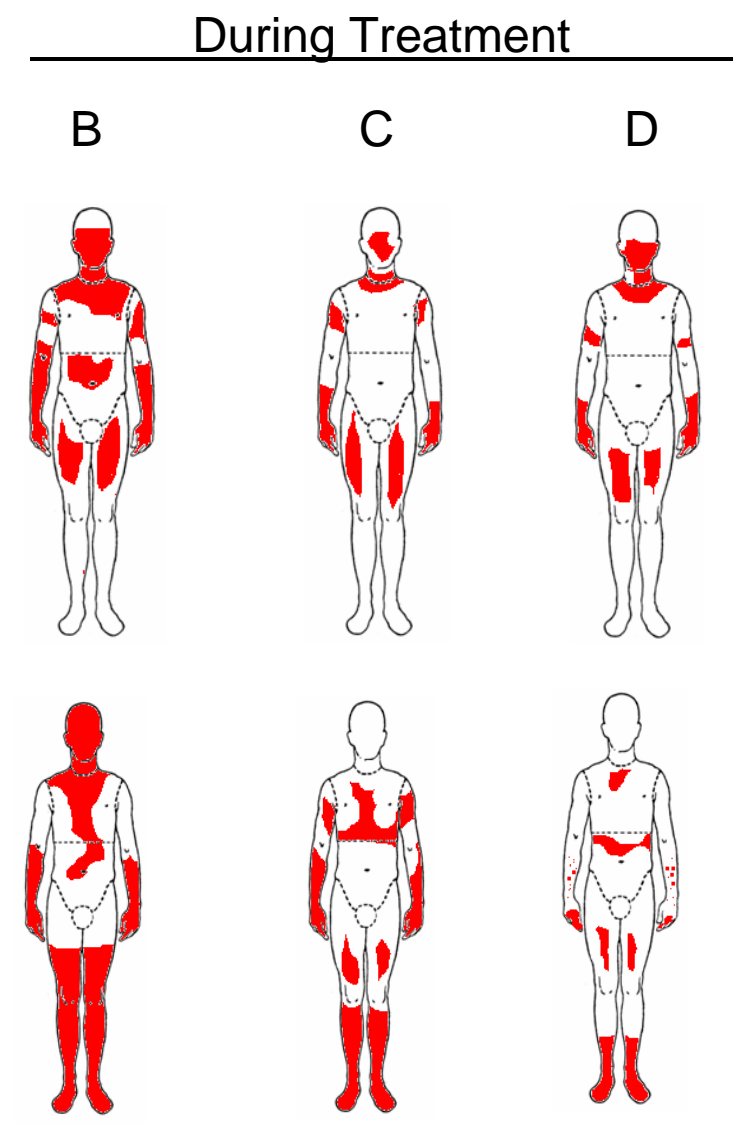

Patient 2

\section{Patient 1}

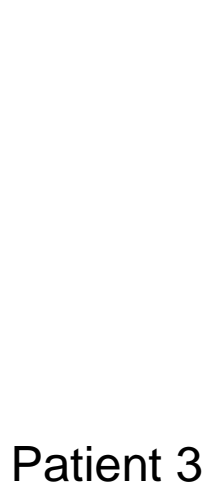

\section{Patient 3}

Figure 2
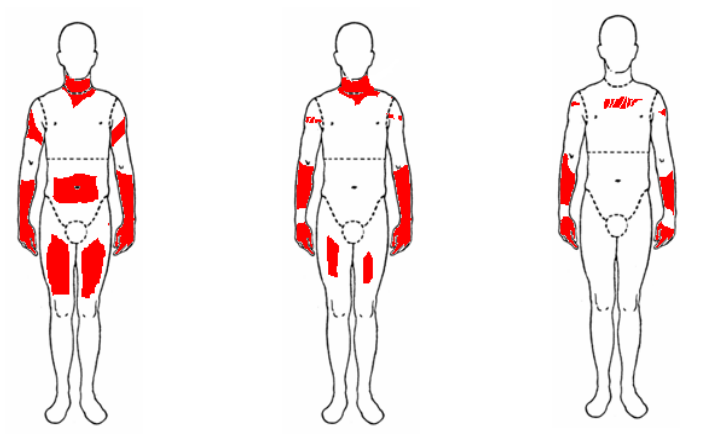


\section{Figure 3}

A

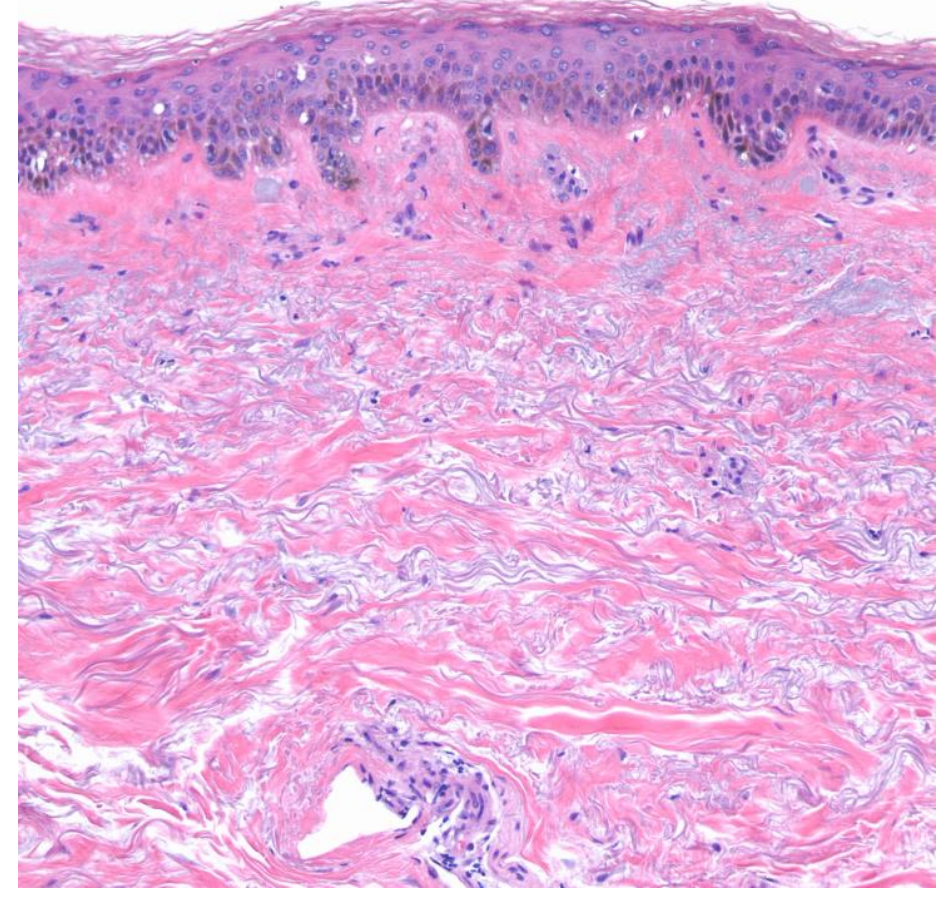

B

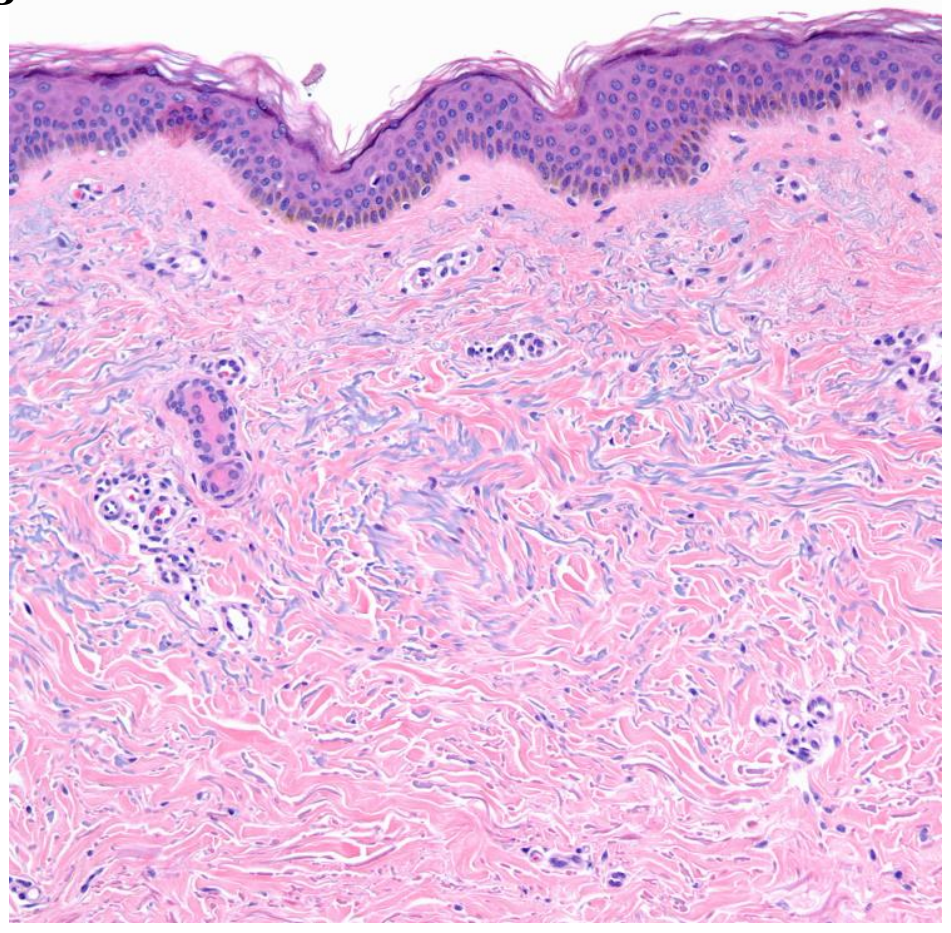

C

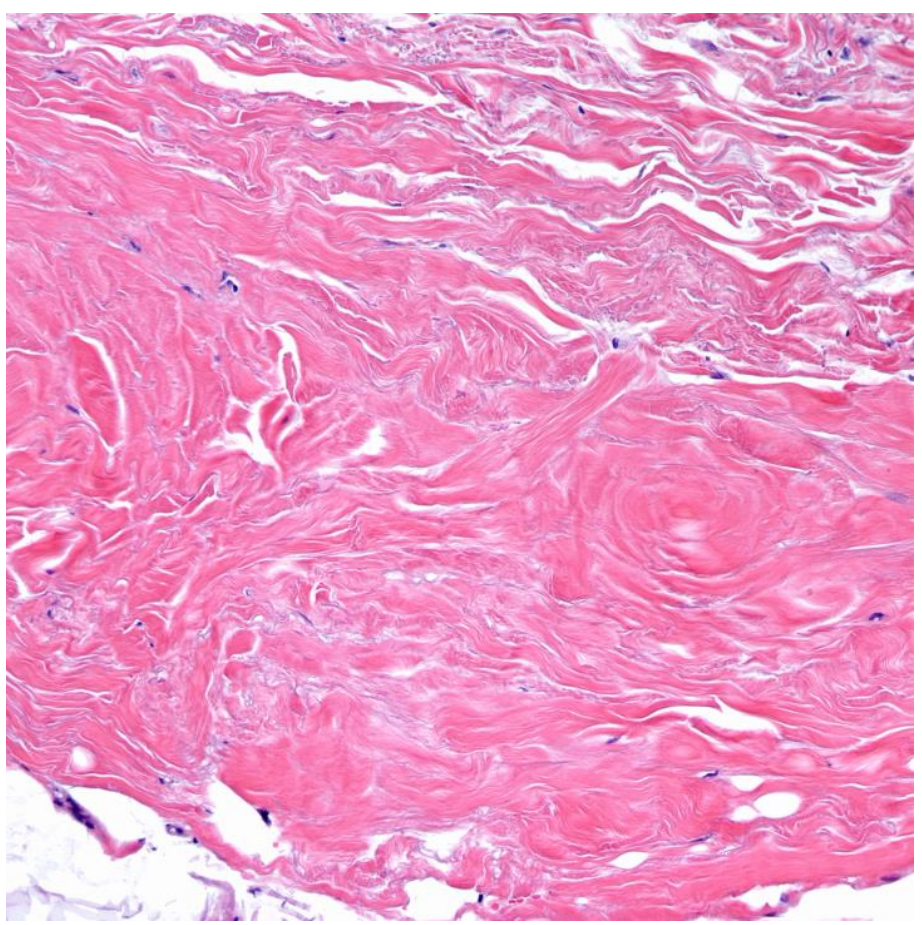

D

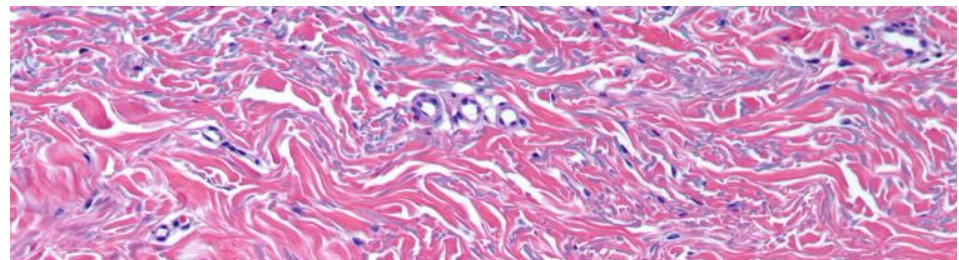
S.

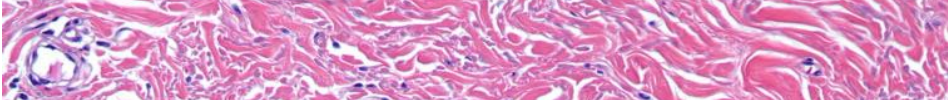

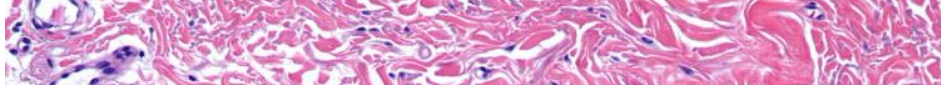

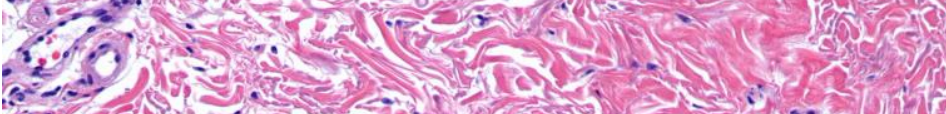

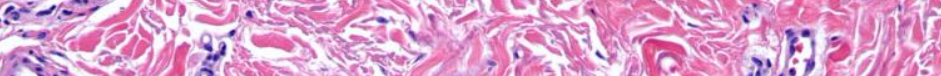

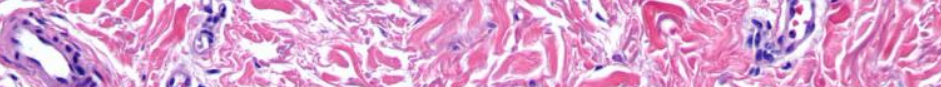

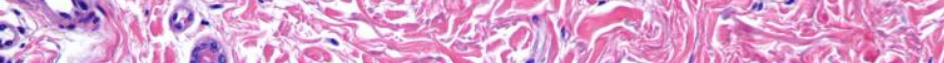

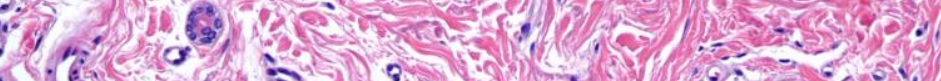
: (2)

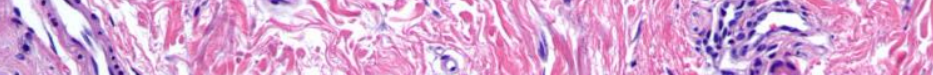

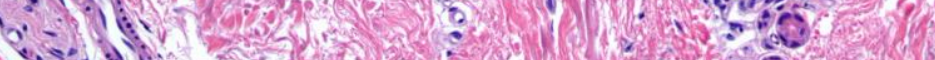
4.

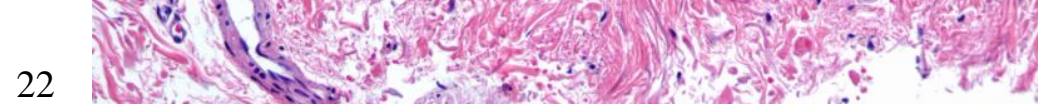




\section{Figure 4}

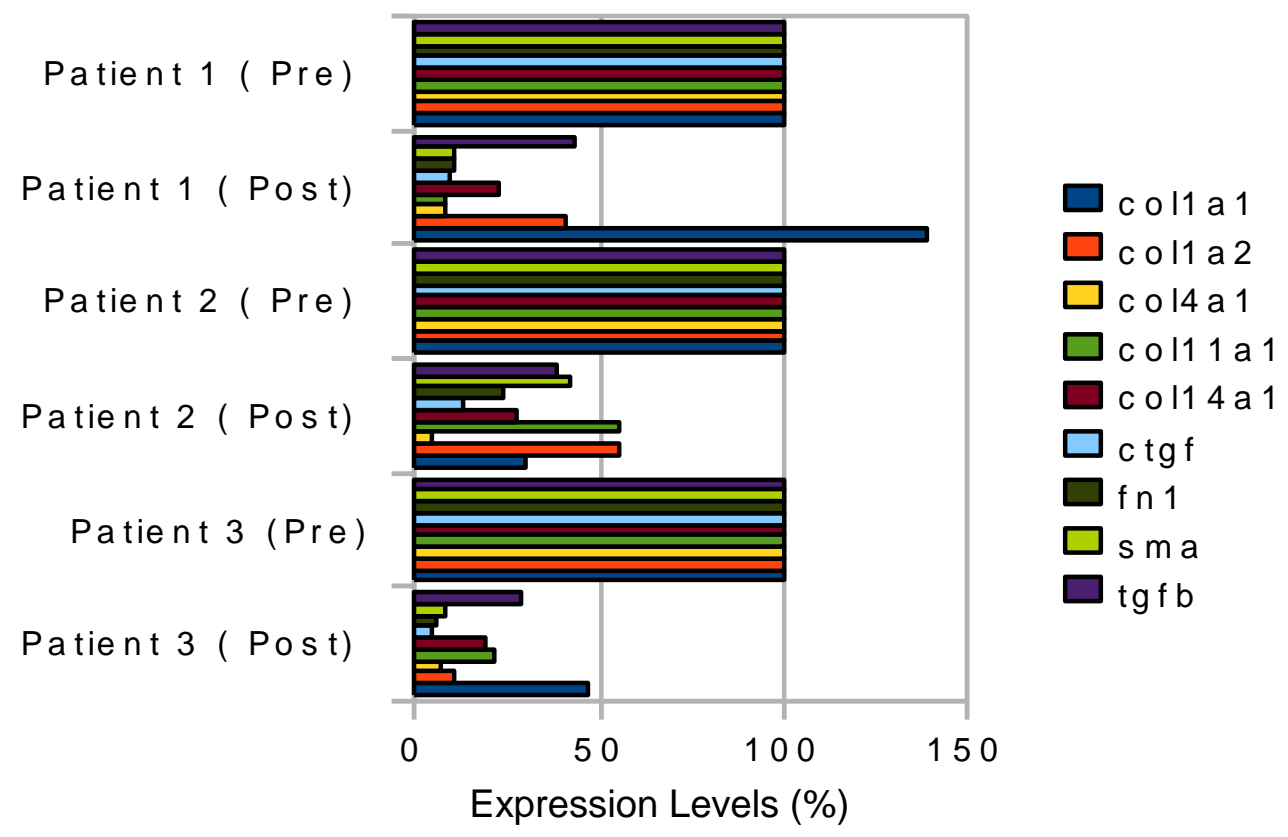

